Trauma Berufskrankh 2009 · 11[Suppl 2]: 130-135

DOI 10.1007/s10039-009-1481-6

Online publiziert: 20. Mai 2009

(c) Springer Medizin Verlag 2009

M.H. Amlang - V. Betz - M. Gavlik · H. Zwipp

Klinik für Unfall- und Wiederherstellungschirurgie, Universitätsklinikum Carl Gustav Carus, TU Dresden

\title{
Ergebnisse der Meniskusrefixation mit Pfeilen
}

\section{Material und Methode}

\section{Literatursuche} messen lassen. Dabei sind die Ergebnisse sowohl hinsichtlich der Heilungsrate als auch der Art und Häufigkeit der Komplikationen zu vergleichen.

Vor einem Jahrzehnt wurden Pfeile zur Meniskusrefixation experimentell untersucht und in die klinische Routine eingeführt $[2,4,10,11,12,38]$. Es handelt sich in der Regel um starre, fadenfreie, resorbierbare Implantate, wobei die Fixationsfestigkeit im Wesentlichen aus der widerhakenartigen Oberflächengestaltung resultiert (• Abb. 1). Diese Implantate werden aufgrund der einfachen Operationstechnik auch in schwerer zugänglichen Regionen wie am Innenmeniskushinterhorn häufig verwendet. Eine weitere Generation von Implantaten wie FasT-fix ${ }^{\otimes}$ oder Rapid-Loc ${ }^{\oplus}$, die als Fadenankersysteme (FAS) zusammengefasst werden können, stehen für den klinischen Einsatz zur Verfügung. In einer ersten, systematischen Literaturanalyse zu den Meniskuspfeilen von Tingart et al. [68] aus dem Jahr 2001 konnte aufgrund der geringen Studienzahl und dem kurzen Nachbeobachtungszeitraum noch keine sichere Beurteilung dieser Technik erfolgen.
Nach einem Jahrzehnt der Anwendung von resorbierbaren Meniskuspfeilen werden in der vorliegenden Arbeit die Ergebnisse dieser Fixationstechnik beim Meniskusriss durch eine systematische Literaturübersicht überprüft.

Studien aus dem angegebenen Zeitraum zur Meniskusrefixation mit Pfeilen, die im Literaturverzeichnis der durchgesehenen Arbeiten gefunden wurden, konnten ebenfalls mit einbezogen werden.

\section{Studienauswahl}

Es wurden klinische Studien ausgewählt, die Angaben über den Erfolg der Meniskusrefixation mit Meniskuspfeilen

Die Datenbank der US-amerikanischen „National Library of Medicine" wurde systematisch nach Studien mit den Begriffen „meniscal repair“ und „meniscus arrow“ durchsucht. Die Suchbegriffe wurden mit einer „und“-Verknüpfung versehen und die Arbeiten nach Angaben zum klinischen Ergebnis analysiert [36]. Weitere Suchbegriffe waren "meniscal dart“, „meniscal fastener“, „clearfix screw“ und „biostinger“. Das „Versagen“ der Fixation bzw. die Heilungsstörung wurden nach Tuckman et al. [69] als Notwendigkeit einer sekundären Meniskusresektion am rekonstruierten Meniskus definiert, wobei die Differenz zu 100\% als „Heilung“ angegeben wurde. Bei eindeutiger Angabe der Heilungsrate in einer Studie wurde diese Zahl berücksichtigt.

Der Beobachtungszeitraum umfasste Studien von August 2000-Januar 2009, da die Untersuchung direkt an die Literaturanalyse von Tingart et al. [68] angefügt werden sollte, deren Beobachtungszeitraum sich von 1966-August 2000 erstreckte.
(- Abb. 1) enthielten. Studien über FASImplantate wie Rapid-Loc ${ }^{\circledR}$ und FasT-fix wurden nicht mit einbezogen.

Aus den Arbeiten wurden Angaben $\mathrm{zu}$

- Studientyp,

- Patientenzahl,

- Patientenalter,

- Fixationsmethode,

- Risslokalisation,

- Risstyp,

- Kniestabilität,

- Nachuntersuchungszeitraum und

- Heilungsrate

erfasst. Die Beurteilung der Autoren zum Einsatz von Meniskuspfeilen wurde den Kategorien „positiv“, „differenziert“ und „unbefriedigend“ zugeordnet. Bei der Kategorie „positiv“ musste eine klare Empfehlung zur Verwendung der Pfeile als gleichwertig zur Naht vorhanden sein. Bei der Kategorie „unbefriedigend“ oder „negativ“ war eine kritische Beurteilung der Meniskuspfeile entweder aufgrund der Versagensrate oder wegen der Komplikationshäufigkeit enthalten. Bei einer „dif- 


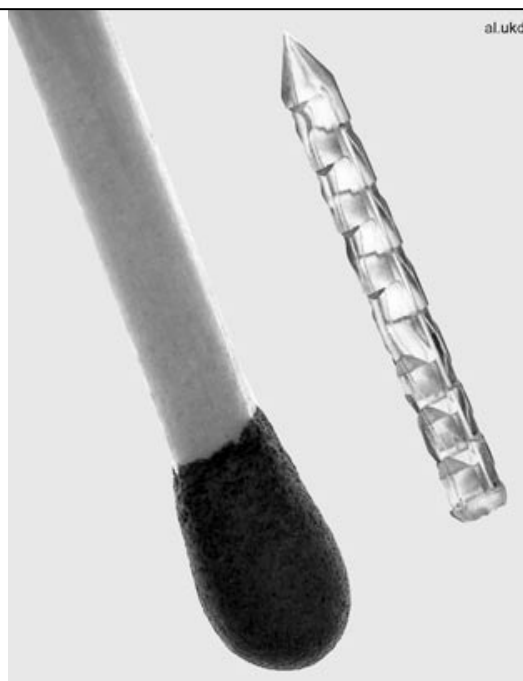

Abb. $1 \Delta$ Meniscal dart ${ }^{\circledR}$ (Foto: Universitätsklinikum Carl Gustav Carus, TU Dresden)

ferenzierten“ Beurteilung konnte die Arbeit nicht in die 2 erstgenannten Kategorien eingeordnet werden.

\section{Ergebnisse}

\section{Studienbeschreibung}

Im Zeitraum August 2000-Februar 2009 konnten unter dem Suchbegriff „meniscal repair" 763 Arbeiten identifiziert werden. Bei der „und“-Verknüpfung mit „arrow“ fanden sich noch 46 Publikationen. Unter Einbeziehung des Literaturverzeichnisses dieser Studien und von Übersichtsarbeiten wurden 19 weitere Arbeiten $\mathrm{zu}$ diesem Thema gefunden, sodass letztlich 65 Artikel ausgewertet wurden.

Nach Durchsicht dieser Publikationen wurden 15 Originalarbeiten gefunden, die über klinische Ergebnisse der Meniskusrefixation mit Pfeilen berichteten (• Tab. 1). Eine Fallzahl unter 20 war ein Ausschlusskriterium [53]. Studien zu spezifischen Implantaten, die zwar ein ähnliches Funktionsprinzip haben, wie die Menikusschraube, wurden aufgrund der geringen Anzahl nicht einbezogen [13, 27]. Weitere 11 Arbeiten enthielten Berichte über implantatspezifische Komplikationen, wobei auch Kasuistiken über Implantate der FAS-Gruppe wie Rapid-Loc ${ }^{\oplus}$ und FasT-fix ${ }^{\oplus}$ gefunden wurden $[5,14,18$, 20, 30, 33, 34, 55, 56, 59, 63, 71]. Bei den übrigen Publikationen handelte es sich um experimentelle Arbeiten oder Über-

Trauma Berufskrankh 2009 • 11 [Suppl 2]: 130-135 DOI 10.1007/s10039-009-1481-6

(c) Springer Medizin Verlag 2009

\section{M.H. Amlang · V. Betz · M. Gavlik · H. Zwipp Ergebnisse der Meniskusrefixation mit Pfeilen}

\section{Zusammenfassung}

Die US-amerikanische „National Library of Medicine" wurde systematisch nach zwischen August 2000 und Januar 2009 veröffentlichten Studien mit den Suchbegriffen "meniscal repair" und "meniscus arrow" durchsucht. Die Beurteilung der Autoren zum Einsatz von Meniskuspfeilen wurde den Kategorien „positiv" , ,differenziert" und ", unbefriedigend" zugeordnet. Es wurden 15 Originalarbeiten gefunden, die über klinische Ergebnisse der Meniskusrefixation mit Pfeilen berichteten. Für den Zeitraum 2002-2004 wurden 6 Studien identifiziert, die alle den Einsatz von Meniskuspfeilen als gleichwertig zur Naht bewerteten. Unter den 9 Arbeiten im Zeitraum 2005-2009 befanden sich 5 mit kritischer, 3 mit differenzierter und 1 mit posi- tiver Bewertung. Die Heilungsraten der Arbeiten vom Zeitraum 2002-2004 von 90\% (87-97\%) sanken im Zeitraum 2005-2009 auf 74\% (59-95\%). Das Follow-up der Studien betrug im Zeitraum 2002-2004 im Durchschnitt 34 Monate, in den Jahren 2005-2009 im Durchschnitt 47 Monate. Die Ergebnisse der Literaturanalyse geben berechtigten Anlass für die Empfehlung, für eine Naht gut zugängliche Meniskusabschnitte nicht mit Meniskuspfeilen, sondern klassisch entweder mit Outside-in- oder Inside-out-Naht zu fixieren.

\section{Schlüsselwörter}

Meniskusriss · Meniskusrefixation · Meniskusnaht $\cdot$ Meniskuspfeil $\cdot$ Arthroskopie

\section{Results of meniscal repair using arrows}

\section{Abstract}

In the period between August 2000 and January 2009 articles were retrieved from the American "National Library of Medicine" in the framework of a systematic search for the key words "meniscal repair" and "meniscus arrow". The authors' opinions on the use of meniscus arrows were categorised as "positive", "differentiated" and "unsatisfactory". In all, 15 publications were found that reported clinical results of meniscal repair using arrows. Six studies were published between 2002 and 2004 which considered arrows and sutures to be equivalent. Of the nine studies published between 2005 and 2009 five studies fell into the category "unsatisfactory", three studies into "differentiated" and one study into "pos- itive". The clinical success rate of 90\% (8797\%) found in the studies published between 2002 and 2004 decreased to $74 \%$ (59-95\%) as published between 2005 and 2009. The average follow-up in the studies published between 2002 and 2004 was 34 months compared to 47 months in the studies published between 2005 and 2009. The results of this literature review indicate that, if meniscal parts are accessible for suture, the conventional outside-in or inside-out suture techniques should be applied instead of arrows.

\section{Keywords}

Meniscus tear $\cdot$ Meniscal repair $\cdot$ Meniscal suture - Meniscus Arrow · Arthroscopy 


\begin{tabular}{|c|c|c|c|c|c|c|c|c|}
\hline $\begin{array}{l}\text { Erstautor, } \\
\text { Jahr }\end{array}$ & $\begin{array}{l}\text { Evidenz- } \\
\text { grad }\end{array}$ & $\begin{array}{l}\text { An- } \\
\text { zahl }\end{array}$ & Implantat & $\begin{array}{l}\text { Follow-up } \\
\text { (Monate) }\end{array}$ & $\begin{array}{l}\text { Nachunter- } \\
\text { suchung }\end{array}$ & $\begin{array}{l}\text { Heilung } \\
\text { [\%] }\end{array}$ & $\begin{array}{l}\text { Beur- } \\
\text { teilung }\end{array}$ & Bemerkungen \\
\hline $\begin{array}{l}\text { Al-Othman [3], } \\
2002\end{array}$ & 4 & 32 & Bionx Arrow ${ }^{\circledast}$ & $25(10-40)$ & Klinik & 87 & Positiv & $\begin{array}{l}\text { 6-mal Re-ASK mit 2-mal Heilungsstörung + } \\
\text { 2-mal partieller Heilungsstörung }\end{array}$ \\
\hline $\begin{array}{l}\text { Ellermann et al. } \\
{[23], 2002}\end{array}$ & 4 & 105 & Bionx Arrow ${ }^{\circledast}$ & $22(24-43)$ & Klinik & 80 & Positiv & $\begin{array}{l}66 \% \text { mit VKB-Ersatz, alle } 21 \text { Patienten mit } \\
\text { Symptomen hatten Heilungsstörungen, } \\
\text { Außenmeniskus heilte signifikant schlechter }\end{array}$ \\
\hline $\begin{array}{l}\text { Gill u. Diduch } \\
\text { [29], } 2002\end{array}$ & 4 & 32 & Bionx Arrow ${ }^{\circledast}$ & $28(18-39)$ & Klinik & 91 & Positiv & $\begin{array}{l}\text { Alle bei VKB-Ersatz, 3-mal Re-ASK mit 3-mal } \\
\text { Heilungsstörungen }\end{array}$ \\
\hline $\begin{array}{l}\text { Jones et al. [40], } \\
2002\end{array}$ & 4 & 38 & Bionx Arrow ${ }^{\circledast}$ & $>2$ Jahre & Klinik & 93 & Positiv & 32\% Weichteilkomplikationen \\
\hline $\begin{array}{l}\text { Petschke et al. } \\
\text { [57], } 2002\end{array}$ & 4 & 29 & Pfeile & $24(12-42)$ & Klinik & 93 & Positiv & Keine relevanten Weichteilprobleme \\
\hline $\begin{array}{l}\text { Steenbrugge et } \\
\text { al. [66], } 2004\end{array}$ & 4 & 35 & Bionx Arrow ${ }^{\circledR}$ & 6 Jahre & Klinik & 97 & Positiv & \\
\hline $\begin{array}{l}\text { Kurzweil et al. } \\
{[46], 2005}\end{array}$ & 4 & 57 & Bionx Arrow ${ }^{\circledast}$ & $54(36-70)$ & Klinik+MRT & 72 & $\begin{array}{l}\text { Unbefrie- } \\
\text { digend }\end{array}$ & $80 \%$ Heilung bei VKB-Ersatz \\
\hline $\begin{array}{l}\text { Sarimo et al. } \\
{[60], 2005}\end{array}$ & 4 & 20 & Bionx Arrow ${ }^{\circledast}$ & $26(6-38)$ & Klinik & 90 & $\begin{array}{l}\text { Differen- } \\
\text { ziert }\end{array}$ & 13-mal Re-ASK, 7-mal Knorpelpathologie \\
\hline $\begin{array}{l}\text { Lee u. Diduch } \\
{[47], 2005}\end{array}$ & 4 & 32 & Bionx Arrow ${ }^{\circledast}$ & 6,6 Jahre & Klinik & 72 & $\begin{array}{l}\text { Unbefrie- } \\
\text { digend }\end{array}$ & $\begin{array}{l}\text { Gleichzeitig VKB-Ersatz, nach 2,3 Jahren 91\% } \\
\text { Heilung }\end{array}$ \\
\hline $\begin{array}{l}\text { Barber u. Coons } \\
{[6], 2006}\end{array}$ & 4 & 41 & BioStinger $^{\circledast}$ & $39(24-69)$ & Klinik & 95 & Positiv & $\begin{array}{l}\text { 6-mal Re-ASK mit 2-mal Heilungsstörung, 4- } \\
\text { mal periphere Migration, 3-mal Entfernung } \\
\text { der Pfeile }\end{array}$ \\
\hline $\begin{array}{l}\text { Tuckman et al. } \\
{[69], 2006}\end{array}$ & 4 & $\begin{array}{l}133 \\
(64)\end{array}$ & $\begin{array}{l}\text { Diverse Tech- } \\
\text { niken, u. a. } \\
\text { Bionx Arrow } \\
(\mathrm{n}=64)\end{array}$ & $>2$ Jahre & Klinik & 65 & $\begin{array}{l}\text { Unbefrie- } \\
\text { digend }\end{array}$ & $\begin{array}{l}\text { Etwa } 50 \% \text { veraltete Risse, Außenmeniskus } \\
\text { heilte signifikant schlechter }\end{array}$ \\
\hline $\begin{array}{l}\text { Siebold et al. } \\
{[62], 2007}\end{array}$ & 4 & 113 & Bionx Arrow ${ }^{\circledast}$ & $>5$ Jahre & Klinik & 72 & $\begin{array}{l}\text { Unbefrie- } \\
\text { digend }\end{array}$ & $\begin{array}{l}66 \% \text { der ASK gleichzeitig mit VKB-Ersatz, } \\
\text { nach 2,8 Jahren nur } 20 \% \text { Heilungsstörung }\end{array}$ \\
\hline $\begin{array}{l}\text { Tielinen et al. } \\
\text { [67], } 2007\end{array}$ & 4 & 77 & Bionx Arrow ${ }^{\circledast}$ & $25(5-56)$ & Klinik+MRT & 66 & $\begin{array}{l}\text { Differen- } \\
\text { ziert }\end{array}$ & $\begin{array}{l}\text { Pfeile nicht für instabile Risse, Meniskus+VKB } \\
79 \% \text { Heilung }\end{array}$ \\
\hline $\begin{array}{l}\text { Bryant et al. } \\
{[17], 2007}\end{array}$ & 1 & 51 & Bionx Arrow ${ }^{\circledast}$ & $28(20-36)$ & Klinik & 78 & $\begin{array}{l}\text { Differen- } \\
\text { ziert }\end{array}$ & $\begin{array}{l}\text { 5-mal intraoperativ Wechsel auf Naht, kein } \\
\text { statistischer Unterschied zur Naht, aber } \\
\text { Knorpelschäden und Fremdkörperreaktion }\end{array}$ \\
\hline $\begin{array}{l}\text { Gifstad et al. } \\
\text { [28], } 2009\end{array}$ & 4 & 118 & Biofix Arrow ${ }^{\circledast}$ & $\begin{array}{l}4,7(1,8- \\
7,7) \text { Jahre }\end{array}$ & ASK & 59 & $\begin{array}{l}\text { Unbefrie- } \\
\text { digend }\end{array}$ & $\begin{array}{l}\text { Zeit zwischen Erst-ASK und Re-ASK } 17 \\
\text { (1-75 Monate) }\end{array}$ \\
\hline
\end{tabular}

sichten $[4,10,11,12,15,19,21,22,25,26,37$, $39,43,48,49,51,52,53,61,64,65,70]$.

Unter den 15 Arbeiten, die in die Analyse einbezogen wurden, war nur eine Studie mit dem Evidenzgrad 1 für ein prospektiv-randomisiertes Design zu finden. Die übrigen Originalarbeiten hatten den Evidenzgrad 4, darunter 4 prospektive Studien.

\section{Studienergebnisse}

Die 15 Arbeiten zur Meniskusrefixation mit Pfeilen erfassten insgesamt 844 Patienten. Die durchschnittliche Heilungsrate betrug $81 \%$, das durchschnittliche Followup 42 Monate. Die Arbeit von Tuckman et al. [69] konnte hier nicht berücksichtigt werden, da nur ein minimales Follow-up von 2 Jahren angegeben wurde. Über eindeutig positive Erfahrungen bei der Verwendung von Meniskuspfeilen berichteten 7 Studien $[3,6,23,29,40,57,66]$. Eine differenzierte Empfehlung für den Einsatz dieser Implantate gaben 3 Arbeiten [17,60, 67]. Über unbefriedigende Erfahrungen mit Meniskuspfeilen berichteten 5 Studien $[28,46,47,62,69]$.

Bei Unterteilung der Arbeiten entsprechend ihres Publikationsdatums in 2 Gruppen entfielen auf den Zeitraum 2002-2004 6 Studien [3, 23, 29, 40, 57, 66]. Alle bewerteten den Einsatz von Meniskuspfeilen als gleichwertig zur Naht. In der Arbeit von Ellermann et al. [23] wurde bereits ausdrücklich auf das Risiko der
Knorpelschädigung hingewiesen. Bei den 9 Arbeiten im Zeitraum 2005-2009 fanden sich 5 Studien mit kritischer [28, 46, $47,62,69], 3$ mit differenzierter $[17,60,67]$ und 1 Studie mit positiver Bewertung [6]. Die angegebenen Heilungsraten der Arbeiten vom Zeitraum 2002-2004 von 90\% (87-97\%) sanken im Zeitraum 2005-2009 auf $74 \%$ (59-95\%). Das Follow-up war bei den Studien im Zeitraum 2002-2004 im Durchschnitt 34 Monate und betrug bei den Arbeiten aus den Jahren 2005-2009 im Durchschnitt 47 Monate.

Unter den 5 Arbeiten mit einem längeren Follow-up als 48 Monaten wies nur die Arbeit von Steenbrugge et al. [66] eine positive Bewertung bei einer Heilungsrate von $97 \%$ auf, wobei diese der ersten Peri- 


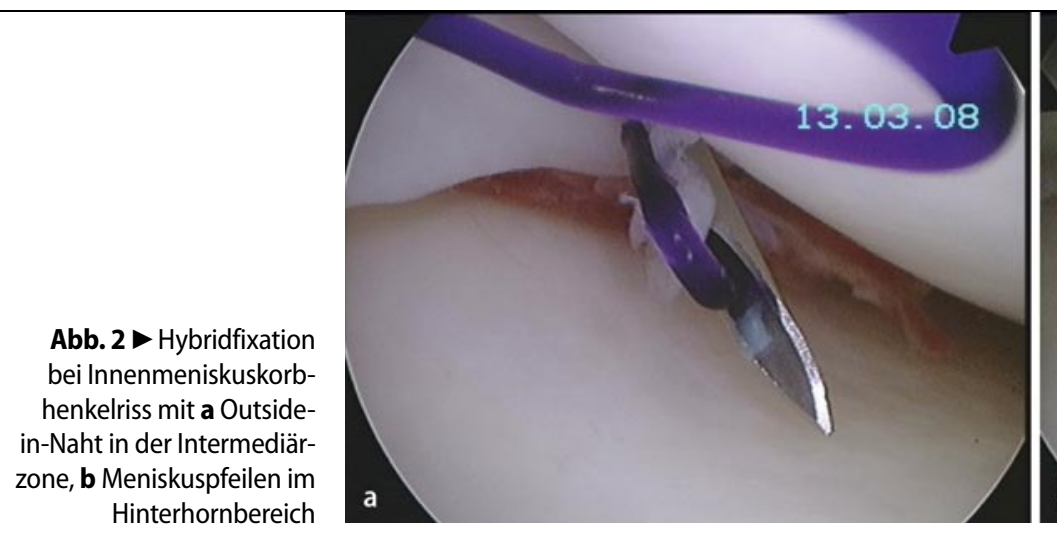

ode zuzuordnen war. Die 4 Arbeiten mit einem Follow-up von mehr als 48 Monaten aus dem Zeitraum 2005-2009 kamen $\mathrm{zu}$ einem unbefriedigenden Ergebnis mit einer durchschnittlichen Heilungsrate von $69 \%[46,47,62]$.

\section{Diskussion}

King [42] konnte bereits 1936 eindrucksvoll zeigen, dass die Heilung des Meniskusrisses v. a. von der Risslokalisation und Rupturmorphologie abhängt. Da die Heilungspotenz eines Meniskusrisses intraoperativ nie absolut sicher zu beurteilen ist, kann auch eine technisch perfekte Fixation keine Garantie für eine Heilung sein. Der Wunsch nach einer sicheren, schnellen, leicht handhabbaren Fixation hat vor reichlich einem Jahrzehnt zur Entwicklung der Meniskuspfeile geführt. Waren die Beurteilungen in den ersten Jahren noch eindeutig positiv, setzte sich im Laufe der Zeit überwiegend eine differenzierte bis kritische Meinung zum Einsatz von Meniskuspfeilen durch. Eine Zäsur für die Einschätzung dieser Implantate waren offensichtlich die Arbeiten von Kurzweil et al. [46] und Lee u. Diduch [47] aus dem Jahr 2005. Eine Ursache für die z. T. unbefriedigenden Ergebnisse und die damit kritischere Einschätzung der Meniskuspfeile dürfte das längere Follow-up bei den Studien der 2. Periode sein. Besonders eindrücklich konnten dies Ellermann et al. [23] und Siebold et al. [62] an ihrem Patientengut zeigen. Die Versagensrate von $20 \%$ nach 2,8 Jahren stieg bei einem Beobachtungszeitraum von 6 Jahren auf $28,4 \%$ an. Die 4 Studien mit einem mittleren Follow-up von mehr als 48 Monaten von 2005-2009 zeigten vergleichbare Ergebnisse. So gaben Gifstad et al.
[28] eine Heilungsrate von 59\%, Kurzweil et al. [46] von $72 \%$, Lee u. Diduch [47] von $72 \%$ und Siebold et al. [62] ebenfalls von $72 \%$ an. In der einzigen Untersuchung mit einem vergleichbaren Follow-up aus der ersten Periode berichteten Steenbrugge et al. [66] bei ihrem Patientengut über eine Heilungsrate von $97 \%$.

Eine Arbeitsgruppe mit einer Publikation im Zeitraum 2005-2009 mit positiver Bewertung der Meniskuspfeile publizierte noch im gleichen Jahr eine Arbeit mit eher kritischer Beurteilung des Rapid-Loc ${ }^{\circledast}$ und 2 Jahre später eine Studie mit positiver Bewertung des FasT-fix ${ }^{\circledast}[6,7,8]$.

Unabhängig von der Zeitperiode wurden durch mehrere Studien bessere Heilungsraten bei Versorgung des Innenmeniskus und gleichzeitigem Ersatz des vorderen Kreuzbandes bestätigt (• Tab. 1, $[23,47,67,69])$. Auf die Bedeutung der Rupturmorphologie für eine erfolgreiche Refixation gingen mehrere Studien ein. In der Arbeit von Tielinen et al. [67] wurden besonders schlechte Ergebnisse mit einer Heilungsrate von nur $38 \%$ bei großen, luxierten Vertikalrissen beobachtet. Allerdings betrug die Heilungsrate insgesamt nur $66 \%$. Bei gleichzeitigem Ersatz des vorderen Kreuzbandes stieg sie auf $79 \%$.

Eine weitere Ursache für die zunehmend differenzierte Beurteilung des Einsatzes von Meniskuspfeilen ist die Zahl der Berichte über implantatspezifische Komplikationen. Dabei verdienen die Beobachtungen von Knorpelschäden an den korrespondierenden Gelenkflächen besondere Aufmerksamkeit [17, 23, 56, 59]. Kasuistiken über derartige Schäden finden sich auch für die Verwendung von fadenbasierten Implantaten der FAS-Gruppe wie dem FasT-fix ${ }^{\oplus}[5,20,30]$, wobei jedoch das Risiko geringer zu sein scheint
[31]. Es sollte aber auch berücksichtigt werden, dass selbst eine Meniskushinterhornresektion aufgrund der anatomischen Gegebenheiten zu einer Knorpelschädigung führen kann $[1,16]$.

Ein implantatspezifisches Problem scheinen Implantatbruch und Pfeilmigration zu sein $[14,33,34]$. Es gibt aber auch für diese Komplikation einzelne Berichte mit Implantaten der FAS-Gruppe [30]. Weitere, mögliche Komplikationen sind die aseptische Synovitis des Kniegelenks und die Ausbildung eines Fremdkörpergranuloms $[45,55,63,71]$.

Die Ergebnisse dieser Arbeit zeigen, dass für die Beurteilung von Innovationen in der Medizin ein ausreichend langer Nachuntersuchungszeitraum erforderlich ist. Die Einführung der Meniskuspfeile ist experimentell und klinisch ausreichend vorbereitet und begleitet worden. Offensichtlich ist ihre Beurteilung sowohl durch die Langzeitergebnisse als auch wegen der Berichte über Fremdkörperreaktionen und Knorpelschäden deutlich kritischer geworden. Ob die neuen Fixationstechniken der FAS-Gruppe langfristig bessere Ergebnisse bringen werden, kann wiederum erst durch die Analyse einer größeren Anzahl von Studien mit ausreichend langem Nachbeobachtungszeitraum beurteilt werden $[32,41,44,50]$. Das Fazit der Untersuchung ist aber nicht die generelle Ablehnung dieser Implantate. Durch die Möglichkeit, diese Technik auch im schlecht zugänglichen Hinterhornbereich einfach, schnell und sicher einzusetzen, ist eine Hybridtechnik mit Fixation der Intermediärzone mit Naht und Fixation des Hinterhorns mit Pfeilen ein guter Kompromiss (• Abb. 2, [16]). Die Ergebnisse der Literaturanalyse geben aber berechtigten Anlass für die Empfehlung, für die 
Naht gut zugängliche Meniskusabschnitte nicht mit Meniskuspfeilen zu fixieren, sondern klassisch entweder in Outsidein- oder Inside-out-Technik zu nähen.

\section{Korrespondenzadresse}

\section{M.H. Amlang}

Klinik für Unfall- und

Wiederherstellungschirurgie,

Universitätsklinikum Carl Gustav Carus,

TU Dresden,

Fetscherstraße 74, 01309 Dresden

Michael.Amlang@uniklinikum-dresden.de

Interessenkonflikt. Der korrespondierende Autor gibt an, dass kein Interessenkonflikt besteht.

\section{Literatur}

1. Agneskirchner JD, Lobenhoffer P (2004) Arthroscopic meniscus surgery: technical-operative methods. Unfallchirurg 107(9):795-801

2. Albrecht-Olsen $P$, Lind $T$, Kristensen $G$, Falkenberg $B$ (1997) Failure strength of a new meniscus arrow repair technique: biomechanical comparison with horizontal suture. Arthroscopy 13(2):183-187

3. Al-Othman AA (2002) Biodegradable arrows for arthroscopic repair of meniscal tears. Int Orthop 26(4):247-249

4. Arnoczky SP, Lavagnino M (2001) Tensile fixation strengths of absorbable meniscal repair devices as a function of hydrolysis time. An in vitro experimental study. Am J Sports Med 29(2):118-123

5. Barber FA (2005) Chondral injury after meniscal repair with RapidLoc. J Knee Surg 18(4):285-288

6. Barber FA, Coons DA (2006) Midterm results of meniscal repair using the BioStinger meniscal repair device. Arthroscopy 22(4):400-405

7. Barber FA, Coons DA, Ruiz-Suarez M (2006) Meniscal repair with the RapidLoc meniscal repair device. Arthroscopy 22(9):962-966

8. Barber FA, Schröder FA, Oro FB, Beavis RC (2008) FasT-Fix meniscal repair: mid-term results. Arthroscopy 24(12):1342-1348

9. Becker R, Fink C (2008) Epidemiologie und Konsequenz von Meniskusverletzungen. Arthroskopie 21:221-222

10. Becker R, Schröder M, Stärke C et al (2001) Biomechanical investigations of different meniscal repair implants in comparison with horizontal sutures on human meniscus. Arthroscopy 17(5):439-444

11. Becker R, Wirz D, Wolf Wolf C et al (2005) Measurement of meniscofemoral contact pressure after repair of bucket-handle tears with biodegradable implants. Arch Orthop Trauma Surg 125(4):254260

12. Bellemans J, Vandenneucker $\mathrm{H}$, Labey L, Van Audekercke R (2002) Fixation strength of meniscal repair devices. Knee $9(1): 11-14$

13. Billante MJ, Diduch DR, Lunardini DJ et al (2008) Meniscal repair using an all-inside, rapidly absorbing, tensionable device. Arthroscopy 24(7):779785

14. Bonshahi AY, Hopgood P, Shepard GJ (2004) Migration of a broken meniscal arrow: a case report and review of the literature. Knee Surg Sports Traumatol Arthrosc 12(1):50-51
15. Borden P, Nyland J, Caborn DN et al (2003) Biomechanical comparison of the FasT-Fix meniscal repair suture system with vertical mattress sutures and meniscus arrows. Am J Sports Med 31(3):374378

16. Bosch U (2005) Stage-based treatment by partial meniscectomy, meniscal refixation and transplantation. Zentralbl Chir 130(4):314-320

17. Bryant D, Dill J, Lichfiled R et al (2007) Effectiveness of bioabsorbable arrows compared with insideout suturing for vertical, reparable meniscal lesions: a randomized clinical trial. Am J Sports Med 35(6):889-896

18. Calder SJ, Myers PT (1999) Broken arrow: a complication of meniscal repair. Arthroscopy 15(6):651652

19. Chang HC, Nyland J, Caborn DN, Burden R (2005) Biomechanical evaluation of meniscal repair systems: a comparison of the meniscal viper repair system, the vertical mattress FasT-Fix Device, and vertical mattress ethibond sutures. Am J Sports Med 33(12):1846-1852

20. Cohen SB, Anderson MW, Miller MD (2003) Chondral injury after arthroscopic meniscal repair using bioabsorbable Mitek Rapidloc meniscal fixation. Arthroscopy 19(7):E24-E26

21. Cohen SB, Boyd L, Miller MD (2007) Vascular risk associated with meniscal repair using Rapidloc versus FasT-Fix: comparison of two all-inside meniscal devices. J Knee Surg 20(3):235-240

22. Durselen L, Schneider J, Galler M et al (2003) Cyclic joint loading can affect the initial stability of meniscal fixation implants. Clin Biomech (Bristol, Avon) 18(1):44-49

23. Ellermann A, Siebold R, Buelow JU, Sobau C (2002) Clinical evaluation of meniscus repair with a bioabsorbable arrow: a 2- to 3-year follow-up study. Knee Surg Sports Traumatol Arthrosc 10(5):289293

24. Fairbank TJ (1948) Knee joint changes after menis cectomy. J Bone Joint Surg Am 30B(4):664-670

25. Farng E, Sherman O (2004) Meniscal repair devices: a clinical and biomechanical literature review. Arthroscopy 20(3):273-286

26. Fisher SR, Markel DC, Koman JD, Atkinson TS (2002) Pull-out and shear failure strengths of arthroscopic meniscal repair systems. Knee Surg Sports Traumatol Arthrosc 10(5):294-299

27. Frosch KH, Fuchs M, Losch A, Stürmer KM (2005) Repair of meniscal tears with the absorbable clearfix screw: results after 1-3 years. Arch Orthop Trauma Surg 125(9):585-591

28. Gifstad T, Grontvedt T, Drogset JO (2007) Meniscal repair with biofix arrows: results after 4.7 years' follow-up. Am J Sports Med 35(1):71-74

29. Gill SS, Diduch DR (2002) Outcomes after meniscal repair using the meniscus arrow in knees undergoing concurrent anterior cruciate ligament reconstruction. Arthroscopy 18(6):569-577

30. Gliatis J, Kouzelis A, Panagopoulos A, Lambuiris E (2005) Chondral injury due to migration of a Mitek RapidLoc meniscal repair implant after successful meniscal repair: a case report. Knee Surg Sports Traumatol Arthrosc 13(4):280-282

31. Haas AL, Schepsis AA, Hornstein J, Edgar CM (2005) Meniscal repair using the FasT-Fix all-inside meniscal repair device. Arthroscopy 21(2):167-175

32. Hantes ME, Zachos VC, Varitimidis SE et al (2006) Arthroscopic meniscal repair: a comparative study between three different surgical techniques. Knee Surg Sports Traumatol Arthrosc 14(12):1232-1237
33. Hartley RC, Leung YL (2002) Meniscal arrow migration into the popliteal fossa following attempted meniscal repair: a report of two cases. Knee 9(1):69-71

34. Hechtman KS, Uribe JW (1999) Cystic hematoma formation following use of a biodegradable arrow for meniscal repair. Arthroscopy 15(2):207-210

35. Hede A, Larsen E, Sandberg H (1992) Partial versus total meniscectomy. A prospective, randomised study with long-term follow-up. J Bone Joint Surg $\mathrm{Br}$ 74(1):118-121

36. Hinterwimmer S, Engelschalk M; Sauerland S et al (2003) Operative or conservative treatment of anterior cruciate ligament rupture: a systematic review of the literature. Unfallchirurg 106(5):374379

37. Hospodar SJ, Schmitz MR, Golish SR et al (2009) FasT-Fix versus inside-out suture meniscal repair in the goat model. Am J Sports Med 37(2):330-333

38. Hurel C, Mertens F, Verdonk R (2000) Biofix resorbable meniscus arrow for meniscal ruptures: results of a 1-year follow-up. Knee Surg Sports Traumatol Arthrosc 8(1):46-52

39. Jani MM, Silva MJ, Gregush RV, Matava MJ (2004) Mechanical properties of bioabsorbable meniscal arrows as a function of tear location: an ex vivo experimental study. Am J Sports Med 32(3):666-674

40. Jones HP, Lemos MJ, Wilk RM et al (2002) Two-year follow-up of meniscal repair using a bioabsorbable arrow. Arthroscopy 18(1):64-69

41. Kalliakmanis $A$, Zourntos $S$, Bousgas D, Nikolaou $P$ (2008) Comparison of arthroscopic meniscal repair results using 3 different meniscal repair devices in anterior cruciate ligament reconstruction patients. Arthroscopy 24(7):810-816

42. King D (1936) The healing of semilunar cartilages. Clin Orthop 252:4-7

43. Kocabey Y, Chang HC, Brand JC Jr et al (2006) A biomechanical comparison of the FasT-Fix meniscal repair suture system and the RapidLoc device in cadaver meniscus. Arthroscopy 22(4):406-413

44. Kotsovolos ES, Hantes ME, Mastrokalos DS et al (2006) Results of all-inside meniscal repair with the FasT-Fix meniscal repair system. Arthroscopy 22(1):3-9

45. Kumar A (2001) Re: Aseptic synovitis after meniscal repair using the biodegradable meniscus arrow in the January 2001 issue by Song et al. Arthroscopy 17(7):792-793

46. Kurzweil PR, Tifford CD, Ignacio EM (2005) Unsatisfactory clinical results of meniscal repair using the meniscus arrow. Arthroscopy 21(8):905

47. Lee GP, Diduch DR (2005) Deteriorating outcomes after meniscal repair using the meniscus arrow in knees undergoing concurrent anterior cruciate ligament reconstruction: increased failure rate with long-term follow-up. Am J Sports Med 33(8):11381141

48. McDermott ID, Richards SW, Hallam P et al (2003) A biomechanical study of four different meniscal repair systems, comparing pull-out strengths and gapping under cyclic loading. Knee Surg Sports Traumatol Arthrosc 11(1):23-29

49. Miller MD, Hart JA (2005) All-inside meniscal repair. Instr Course Lect 54:337-340

50. Miller MD, Kline AJ, Gonzales J, Beach WR (2002) Pitfalls associated with FasT-Fix meniscal repair. Arthroscopy 18(8):939-943

51. Naqui SZ, Thiryayi WA, Hopgood P, Ryan WG (2005) A biomechanical comparison of the Mitek RapidLoc, Mitek meniscal repair system, clearfix screws and vertical PDS and Ti-Cron sutures. Rev Chir Orthop Reparatrice Appar Mot 91(S8):73 
52. Naqui SZ, Thiryayi WA, Hopgood P, Ryan WG (2006) A biomechanical comparison of the Mitek RapidLoc, Mitek Meniscal repair system, clearfix screws and vertical PDS and Ti-Cron sutures. Knee 13(2):151-157

53. Nierenberg G, Rothem D, Falah M et al (2005) The treatment of meniscal tear with bio-absorbable arrow - 3 years follow-up. Harefuah 144(8):540-543, 599

54. Nyland J, Chang H, Kocabey Y et al (2008) A cyclic testing comparison of FasT-Fix and RapidLoc devices in human cadaveric meniscus. Arch Orthop Trauma Surg 128(5):489-494

55. Oliverson TJ, Lintner DM (2000) Biofix arrow appearing as a subcutaneous foreign body. Arthroscopy 16(6):652-655

56. Otte S, Klinger HM, Beyer J, Baums MH (2002) Complications after meniscal repair with bioabsorbable arrows: two cases and analysis of literature. Knee Surg Sports Traumatol Arthrosc 10(4):250-253

57. Petsche TS, Selesnick H, Rochman A (2002) Arthroscopic meniscus repair with bioabsorbable arrows. Arthroscopy 18(3):246-253

58. Rankin CC, Lintner DM, Noble PC et al (2002) A biomechanical analysis of meniscal repair techniques. Am J Sports Med 30(4):492-497

59. Ross G, Grabill J, McDevitt E (2000) Chondral injury after meniscal repair with bioabsorbable arrows. Arthroscopy 16(7):754-756

60. Sarimo J, Rantanen J, Tarvainen T et al (2005) Evaluation of the second-generation meniscus arrow in the fixation of bucket-handle tears in the vascular area of the meniscus. A prospective study of 20 patients with a mean follow-up of 26 months. Knee Surg Sports Traumatol Arthrosc 13(8):614618

61. Seil R, Rupp S, Jurecka C et al (2003) Biodegradable meniscus fixations: a comparative biomechanical study. Rev Chir Orthop Reparatrice Appar Mot 89(1):35-43

62. Siebold R, Dehler C, Boes L et al (2007) Arthroscopic all-inside repair using the meniscus arrow: long-term clinical follow-up of 113 patients. Arthroscopy 23(4):394-399

63. Song EK, Lee KB, Yoon TR (2001) Aseptic synovitis after meniscal repair using the biodegradable meniscus arrow. Arthroscopy 17(1):77-80

64. Staerke C, Bochwitz C, Groebel KH et al (2004) The effect of meniscus compression on the biomechanical properties of repaired meniscal lesions. Winner of the AGA-DonJoy Award 2003. Arch Orthop Trauma Surg 124(4):221-225

65. Staerke C, Kopf S, Becker R (2008) The extent of laceration of circumferential fibers with suture repair of the knee meniscus. Arch Orthop Trauma Surg 128(5):525-530

66. Steenbrugge F, Verdonk R, Hürel C, Verstraete K (2004) Arthroscopic meniscus repair: inside-out technique vs. Biofix meniscus arrow. Knee Surg Sports Traumatol Arthrosc 12(1):43-49

67. Tielinen L, Lindahl J, Koskinen S, Hirvensalo E (2007) Clinical and MRI evaluation of meniscal tears repaired with bioabsorbable arrows. Scand J Surg 96(3):252-255

68. Tingart M, Höher J, Bouillon B, Tiling (2001) Meniscus refixation: suture or anchor? Unfallchirurg 104(6):507-512

69. Tuckman DV, Bravman JT, Lee SS et al (2006) Outcomes of meniscal repair: minimum of 2-year follow-up. Bull Hosp Jt Dis 63(3-4):100-104
70. Walsh SP, Evans SL, O'Doherty DM, Barlow IW (2001) Failure strengths of suture vs. biodegradable arrow and staple for meniscal repair: an in vitro study. Knee 8(2):151-156

71. Yoo JH, Yoon JR, Lee SJ (2008) Parameniscal cyst formation after arthroscopic meniscal repair with biodegradable meniscal arrow: a case report. Knee Surg Sports Traumatol Arthrosc 16(9):815-817

72. Zantop TEggers Ak, Musahl V et al (2005) Cyclic testing of flexible all-inside meniscus suture anchors: biomechanical analysis. Am J Sports Med 33(3):388-394 